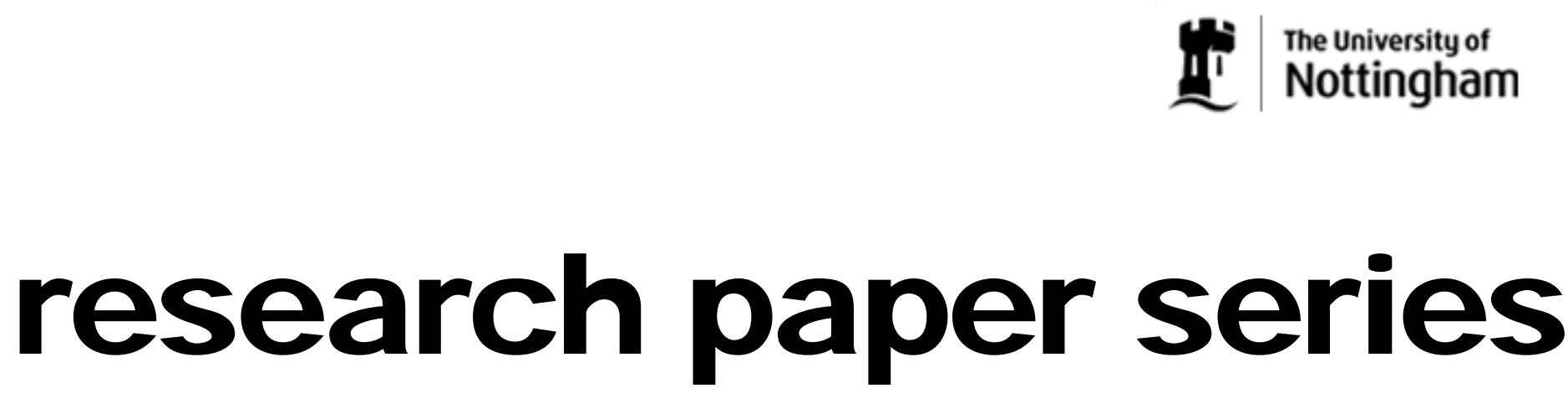

Theory and Methods

Research Paper 2007/23

Trade liberalization and the spatial distribution

of economic activity within a country

by

Pedro Moncarz and Michael Bleaney

The Centre acknowledges financial support from The Leverhulme Trust under Programme Grant F114/BF

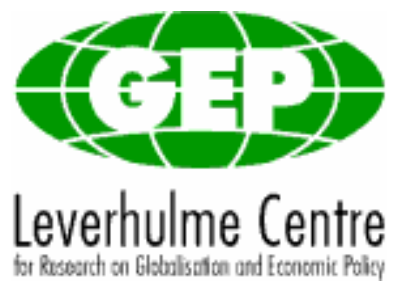




\section{The Authors}

Pedro Moncarz is an Assistant Research at the National University of Cordoba (Argentina).

Michael Bleaney is a Professor of Economics at the University of Nottingham.

\section{Acknowledgements}

We thank Frédéric Robert-Nicoud, Peter Wright and seminar participants at the University of Antwerp (Belgium), University of Nottingham (UK) and National University of Córdoba (Argentina) for valuable comments and suggestions. The usual disclaimer applies. Pedro Moncarz is grateful to The Leverhulme Trust for financial support under Programme Grant F114/BF. Financial support from the EULALIA Red under the ALFA II Programme of the European Union (contract $N^{\circ}$ AML/B7-311-97/0666/II-0058-FA) is also acknowledged. 


\title{
Trade liberalization and the spatial distribution \\ of economic activity within a country
}

\author{
by
}

\author{
Pedro Moncarz and Michael Bleaney
}

\begin{abstract}
The effects of the liberalization of international trade are analyzed in a New Economic Geography model of a country with an asymmetric distribution of housing between regions. Labour is mobile between regions but not between countries. Trade liberalization tends to reduce inequalities in the distribution of population between the two regions, although population is always more unequally distributed than housing. Results are similar when there is a bias in preferences towards home-produced varieties of manufactures. If consumers care relatively little about housing and transport costs are high enough, an agglomerated equilibrium becomes stable.
\end{abstract}

JEL classification: F12, R12

Keywords: International trade, agglomeration, economic geography, housing costs

\section{Outline}

1. Introduction

2. The model

3. Characterizing the equilibrium

4. Trade liberalization

5. Consumption bias

6. Summary and conclusions 


\section{Non-Technical Summary}

Most of the literature dealing with the geographical effects of trade liberalization considers that each country engaged in the process is internally homogeneous (e.g. Krugman, 1991; Krugman and Venables, 1995 and 1996; Venables, 1996; Helpman, 1998). This paper addresses the effects on different regions of a country of a reduction in that country's barriers to external trade. Does the promotion of trade intensify or reduce regional disparities inside the country? This question acquires more importance when we look at the behaviour of some developing countries that since the middle of the 1970s, and mostly during the 1990s, adopted policies more oriented to the external markets.

The approach we follow is to extend the two-region model of Helpman (1998), in which consumers' utility depends on the consumption of manufactures, which are produced under increasing returns to scale, and on the consumption of a non-traded good whose supply is fixed and immobile ("housing"). A significant advantage of this model is that it can easily incorporate asymmetries between the regions, by allowing the distribution of housing to be unequal, which is more realistic than the usual symmetric model. We follow the route pioneered by Krugman and Livas Elizondo (1996) of adding to the model a third region (the rest of the world or ROW), trade with which involves costs at least as high as between domestic regions.

We find is that, except in certain cases when inter-regional transport costs are very high, reducing barriers to international trade tends to reduce the inequality in the distribution of population between the two regions (although it always remains more unequally distributed than the stock of housing). This effect arises because the consumption of home manufactures becomes less important relative to the consumption of housing when there is more trade with the rest of the world.

If consumers have a bias in favour of manufactures produced domestically, the qualitative results remain unchanged. However, in the presence of consumption biases, the effect of trade liberalization on regions' size tends to be larger except in the case of extreme preferences for home-produced manufactures. This happens because changes in trade protection matter very little when consumers' desire for imported varieties shrinks to zero. 


\section{Introduction}

Does the promotion of international trade intensify or reduce regional disparities inside the country?

The standard theoretical approach to this issue builds on the New Economic Geography (NEG) model introduced by Krugman (1991), in which there is a tension between centripetal and centrifugal forces. Centripetal forces arise from increasing returns to scale in one sector (manufacturing) combined with transport costs between regions/countries. Centrifugal forces arise either from an immobile factor of production in another sector (agriculture) that employs an exogenously given proportion of the labour force, as in Krugman and Venables (1995), or from congestion or commuting costs that rise with regional population Fujita et al. (1999), Alonso-Villar (2001) and Murata and Thisse (2005).

These alternative ways of modelling centrifugal forces produce opposite conclusions about the effect of the liberalization of the effect of international trade on the regional concentration of economic activity. Monfort and Nicolini (2000), Paluzie (2001), Crozet and Koening-Soubeyran (2002) all use models with an immobile factor of production, and find that trade liberalization increases regional concentration. Alonso-Villar (2001) considers a model with congestion costs, and finds the opposite result.

The difference in the results arising from the two ways dispersion forces are generated is clearly explained by Alonso-Villar (2001), "The two centrifugal forces, congestion costs and the immobile demand represented by farmers, have different effects on concentration and it should be emphasised that the effects of other parameters, such as transport, can differ depending on the kind of centrifugal force one considers. By considering immobile farmers, concentration is more likely when transport costs are low, because in that case firms do not increase their benefits by moving closer to the dispersed farmers. Conversely, by considering congestion costs, when transport costs between locations decrease concentration is more difficult, since more citizens will want to move to a smaller city where congestion is lower, without paying much for transport costs when delivering goods".

This paper also considers a model with congestion costs. It develops the tworegion model of Helpman (1998), in which consumers' utility depends partly on the consumption of a non-traded good whose supply is fixed and immobile ("housing"). An advantage of this model is that it can easily incorporate asymmetries between the regions, by allowing the distribution of housing to be unequal, which is more realistic than the usual symmetric model. We follow the route pioneered by Krugman and Livas Elizondo (1996) of adding to the model a third region (the rest of the world or ROW), trade with which involves 
costs at least as high as between domestic regions. What we find is that, except in certain cases when inter-regional transport costs are very high, reducing barriers to international trade tends to reduce the inequality in the distribution of population between the two regions (although it always remains more unequally distributed than the stock of housing). This effect arises because the consumption of home manufactures becomes less important relative to the consumption of housing when there is higher consumption of manufactured imports. We also consider an extension of the model in which consumers have a preference for home manufactures.

\section{The Model}

Let us assume that the world is composed of three geographical areas (1, 2, and 3$)$, with Regions 1 and 2 belonging to the same country (the domestic economy) and Region 3 playing the role of the ROW. In each region consumers have a two-tier utility function. The upper tier, which takes a Cobb-Douglas form, determines the consumers' division of expenditure between manufactured goods and housing services. The second tier takes the usual "love for variety" form (Spence, 1976; Dixit and Stiglitz, 1977), and dictates consumer preferences over manufactured varieties. Manufactured goods are traded internally (between Regions 1 and 2) and internationally (between Regions 1 and 2 and the ROW). Trade between domestic regions is subject to transport costs that take the wellknown Samuelson iceberg form ${ }^{1}$. In the case of trade with the ROW, exports to the ROW are frictionless, while imports are subject to iceberg costs that cannot be lower than those for inter-regional trade. ${ }^{2}$ Manufactured varieties are produced using only labour, with the sector being organized as a monopolistic competitive market, and production exhibits increasing returns to scale. Housing services are not tradable, with the supply in each region being fixed. Finally, labour is mobile between domestic regions but immobile internationally. All of these assumptions are similar to those of Helpman (1998), except that in our model the country that reduces trade barriers on imports, the domestic economy, is composed of two (possibly asymmetric) regions. In Helpman (1998) the two countries are internally homogeneous.

\subsection{Consumers}

Consumers' utility in region $i$ takes the following form:

$u_{i}=q_{i}^{\beta} m_{i}^{1-\beta} \quad 0<\beta<1$ 
where $q_{i}$ is the per capita consumption of housing, and $m_{i}$ makes reference to the CES composite of manufactured varieties. In particular, $m_{i}$ is given by:

$$
m_{i}=\left[\sum_{k \in n} c(k)_{i}^{\alpha}\right]^{\frac{1}{\alpha}} \quad 0<\alpha<1
$$

where $c(k)_{i}$ is the per capita consumption of variety $k$ in Region $i$, and $n$ is the mass or number of available varieties produced by the three regions $\left(n=n_{1}+n_{2}+n_{3}\right)$. Under this specification, the elasticity of substitution between any two varieties, as well as the elasticity of demand of each variety (for $n$ large enough), is:

$$
\varepsilon=\frac{1}{1-\alpha}>1
$$

The Cobb-Douglas utility function, together with the CES function for $m_{i}$, means total consumption of each manufactured variety $k$ in Region $i$ is equal to:

$C(k)_{i}=\frac{\left(p(k)_{i}^{j}\right)^{-\varepsilon}}{\left(P_{m i}\right)^{1-\varepsilon}}(1-\beta) E_{i}$

where $p(k)_{i}^{j}$ is the consumer price in Region $i$ of variety $k$ produced in Region $j, E_{i}$ is total expenditure in Region $i$, and $P_{m i}$ is the price index of the differentiated goods in Region $i$.

Trade between Regions 1 and 2 is subject to iceberg costs equal to $t \geq 1$, while imports from the ROW incur costs equal to $\tau$, with $\tau \geq t \geq 1$, which implies the following relationships between producer $p(k)_{j}$ and consumer $p(k)_{i}^{j}$ prices: $^{3,4}$

$$
\begin{array}{ll}
p(k)_{i}^{j}=p(k)_{j} & \text { for } i=j \\
p(k)_{i}^{j}=t p(k)_{j} & \text { for } i \neq j, \quad i, j=1,2 \\
p(k)_{i}^{3}=\tau p(k)_{3} & \text { for } i=1,2 \\
p(k)_{3}^{j}=p(k)_{j} & \text { for } j=1,2
\end{array}
$$

\subsection{Producers}

Production of each manufactured variety uses only labour, and is subject to increasing returns to scale. More specifically, the demand for labour by each firm in Region $i$ to produce variety $k$ is:

$l(k)_{i}=f+v c x(k)_{i}$ 
where $x(k)_{i}$ is the quantity produced of variety $k$ by a firm located in region $i$, while $f>0$ and $v c>0$ are, respectively, the fixed and variable requirements of labour. The existence of fixed costs gives rise to increasing returns to scale, so each variety $k$ is produced just by a single firm, because it is not profitable for two or more firms to produce the same variety.

Assuming firms seek to maximize profits, the producer price of each variety produced in Region $i$ is equal to:

$p(k)_{i}=v c\left(\frac{\varepsilon}{\varepsilon-1}\right) w_{i}$

where $w_{i}$ is the wage rate in Region $i$. Choosing units such that $v c=\frac{\varepsilon-1}{\varepsilon}$, we have:

$p_{i}=w_{i}$

As we can see, all firms located in the same region set the same price. Assuming free entry and exit of firms such that in equilibrium firms achieve zero profit $\left(p_{i} x_{i}-l_{i} w_{i}=0\right)$ means:

$x_{i}=\bar{x}=\frac{f}{v c}(\varepsilon-1)$

Choosing units such that $f=1 / \varepsilon$, we have:

$\bar{x}=1$

As equation (7) shows, the scale of production is constant and identical for all firms independently of where they are located. Assuming full employment we have:

$$
L_{i}=(f+v c \bar{x}) n_{i} \Rightarrow n_{i}=\frac{L_{i}}{f+v c}=L_{i}
$$

\subsection{Equilibrium conditions}

The equilibrium condition for each manufactured variety produced either in Region 1 or 2 requires supply and demand to be equal. For Region 1 we have:

$$
\bar{x}=\frac{p_{1}^{-\varepsilon}}{P_{m 1}^{1-\varepsilon}}(1-\beta) E_{1}+\frac{t\left(t p_{1}\right)^{-\varepsilon}}{P_{m 2}^{1-\varepsilon}}(1-\beta) E_{2}+\frac{p_{1}^{-\varepsilon}}{P_{m 3}^{1-\varepsilon}}(1-\beta) E_{3}
$$

where the terms on the right hand side are, respectively, the total demand (including the quantity that melts in transit), by consumers of Regions 1, 2 and 3, of each variety produced in Region 1. Similar conditions hold for Regions 2 and 3. In condition (9) $P_{m i}$ is 
the manufactured price index for region $i$, which is a decreasing function of the number of varieties locally produced:

$$
\begin{aligned}
& P_{m i}=\left[n_{i} p_{i}^{1-\varepsilon}+n_{j}\left(t p_{j}\right)^{1-\varepsilon}+n_{3}\left(\tau p_{3}\right)^{1-\varepsilon}\right]^{1 /(1-\varepsilon)} \quad i \neq j \text { and } i, j=1,2 \\
& P_{m 3}=\left[n_{1} p_{1}^{1-\varepsilon}+n_{2} p_{2}^{1-\varepsilon}+n_{3} p_{3}^{1-\varepsilon}\right]^{1 /(1-\varepsilon)}
\end{aligned}
$$

Assuming that housing is owned by absentee landlords ${ }^{5}$, total expenditure by residents of Region $i$ is equal to:

$$
E_{i}=\frac{w_{i} L_{i}}{1-\beta} \quad i, j=1,2,3
$$

With labour being mobile between domestic regions, we have that in equilibrium real incomes in Regions 1 and 2 must be equal. For the Cobb-Douglas utility function, the consumer price index $\left(P_{u i}\right)$ is given by:

$P_{u i}=\frac{P_{h i}^{\beta} P_{m i}^{1-\beta}}{\beta^{\beta}(1-\beta)^{(1-\beta)}}$

where $P_{h i}$ is the price of housing services in Region $i$.

Assuming the total stock of housing in the domestic economy is $\mathrm{H}$, and a share $h_{1}$ is located at Region 1, the equilibrium of the housing markets means:

$$
P_{h 1}=\frac{\beta E_{1}}{h_{1} H} \quad \text { and } \quad P_{h 2}=\frac{\beta E_{2}}{\left(1-h_{1}\right) H}
$$

Using (13), the indirect utility function in Region $i$ is given by the real wage in $i$ :

$$
V_{i}=\frac{w_{i}}{P_{u i}}
$$

In equilibrium, and if the two domestic regions have a positive population, we have:

$\bar{V}=V_{1}-V_{2}=w_{1} / P_{u 1}-w_{2} / P_{u 2}=0$

If for a given population distribution indirect utilities are not equalized across domestic regions $(\bar{V} \neq 0)$, migration is governed by the following ad hoc migration equation (Baldwin, et al., 2003):

$$
\Delta s_{1}=\left(V_{1}-V_{2}\right) s_{1}\left(1-s_{1}\right)
$$




\section{Characterizing the equilibrium ${ }^{6}$}

\subsection{A symmetric distribution of housing}

Because any difference in real wages between the regions stimulates migration, there are two types of equilibrium: where the real wages are equal in the two regions, or where the whole of the population is in one region, but any person moving to the other region would receive a lower real wage. When the distribution of housing is symmetric, a symmetric distribution of labour is always an equilibrium, but it is not always a stable equilibrium. Stability can be tested by solving for the relative wage in the two regions as a function of the distribution of labour, and plotting the result. If the region with a slightly larger labour force has a higher (lower) real wage, the symmetric equilibrium is unstable (stable).

As is the case with Helpman's model, the share of income expended on housing $(\beta)$ and the elasticity of substitution between varieties $(\varepsilon)$ are critical parameters. A high elasticity of substitution means that consumers care less about the available number of manufactured varieties, and a high $\beta$ means that housing has a greater weight in their preferences. The outcome differs according to whether the product of these two parameters is high $(\beta \varepsilon>1)$, in which case consumers care strongly about housing, or whether the product is low $(\beta \varepsilon<1)$ and housing is relatively unimportant.

When consumers care strongly about housing $(\beta \varepsilon>1)$, the simulations show that the symmetric equilibrium is always stable, whatever the level of transport costs ( $t$ and $\tau$ ) (Figure 1). When $L_{1}<L_{2}\left(s_{1}<0.5\right)$, then Region 1 always has a higher wage, inducing migration that raises $L_{1}$ and reduces $L_{2}$. Murata and Thisse (2005) obtain a similar result. In their case, dispersion forces are generated by congestion costs rather than by a fixed supply of housing. If congestions costs are high enough and manufactured varieties are close substitutes (high $\varepsilon$ ), a dispersed equilibrium is stable unless transport costs are too high. The reason for this is that for a high elasticity of substitution consumers benefit little from access to a large number of varieties, so the negative effect of increasing commuting costs as population agglomerates in a single region more than compensates for the lower transport costs of having a larger number of varieties locally produced.

In the opposite case, when $\beta \varepsilon<1$, the symmetrical equilibrium is stable only when transport costs are relatively low (Figure 2). If inter-regional transport costs are very low ( $t$ $=1.1$ ), the symmetrical equilibrium is stable for all values of international transport costs. Conversely, if inter-regional transport costs are very high $(t=50)$, the symmetrical equilibrium is always unstable, and the stable equilibrium has $100 \%$ of the population in 
one region or the other. ${ }^{7}$ In the intermediate case shown $(t=2.3)$, the symmetrical equilibrium is stable for free $\operatorname{trade}^{8}(\tau=2.3)$, but not for higher values, and for most values of $\tau$ there are two stable equilibriums with an unequal distribution of population in the two regions.

The difference between the two cases is that, when $\beta \varepsilon<1$, consumers care mainly about consumption of more varieties of manufactures, and are willing to sacrifice a lot of housing to achieve that by living in the more populated region.

\subsection{An asymmetric distribution of housing}

Now let us assume that Region 1 always has more housing than region $2\left(h_{1}>1 / 2\right)$ Figure 3 shows the outcomes for $h_{1}=0.6$, for three values of inter-regional trade costs $(t$ $=1.1,2.3$ and 50), when $\beta \varepsilon>1$. As was the case when $h_{1}=1 / 2$ (Figure 1), the equilibrium is always stable, but now the share of population in Region 1 is always greater than 0.6 , and increases with $t$, because higher inter-regional transport costs make it harder to import extra varieties of manufactures from the other region.

Figure 4 shows the case where $\beta \varepsilon<1$, again with $h_{1}=0.6$, which corresponds to Figure 2 for the symmetric case. As in the symmetric case, a stable equilibrium with high $t$ is where the whole population is agglomerated in one region. With low $t$, there is a stable equilibrium with the population distributed approximately in proportion to the distribution of housing, whatever the level of international trade costs. With $t=2.3$ the picture is somewhat more complicated. A stable but strongly asymmetric equilibrium $\left(s_{1}>0.8\right)$ arises under relatively low barriers to international trade. At high $\tau$, there is a stable equilibrium where Region 2 is empty of population and another stable equilibrium where Region 1 is either empty or has a very small population (this is a consequence of the asymmetric distribution of housing).

An interesting result that emerges under an asymmetric distribution of housing is that the dispersed equilibrium, either stable or unstable, means population is distributed differently from that of housing. This result can be proved as follows. In first place, we have that a symmetric distribution of population cannot be an equilibrium, for $L_{1}=L_{2}$, nominal wages are identical in both regions such that the price index of manufactures is also the same $\left(P_{m 1}=P_{m 2}\right)$. However, since $H_{1}>H_{2}$, the price of housing is cheaper in region 1 $\left(P_{h 1}<P_{h 2}\right)$, this means $V_{1}-V_{2}>0$ for $L_{1}=L_{2}$. Then, with an asymmetric distribution of housing, population must be also asymmetrically distributed in equilibrium. Two 
possibilities arise, $L_{1}>L_{2}$ or $L_{1}<L_{2}$. For any population distribution $\left(L_{1}, L_{2}\right)$ to be an equilibrium we need $\frac{V_{1}}{V_{2}}=\frac{w_{1}}{w_{2}}\left(\frac{P_{h 2}}{P_{h 1}}\right)^{\beta}\left(\frac{P_{m 2}}{P_{m 1}}\right)^{1-\beta}=1$. Using (14), and the fact that in the larger region the nominal wage is higher, if $L_{1}>L_{2}$ is an equilibrium it means $\frac{P_{h 1}}{P_{h 2}}>\left(\frac{w_{1}}{w_{2}}\right)^{1 / \beta}$. On the other hand, if $L_{1}<L_{2}$ is an equilibrium, we must have $\frac{P_{h 1}}{P_{h 2}}<\left(\frac{w_{1}}{w_{2}}\right)^{1 / \beta}$. Given the assumption that housing stock is larger in region 1 than in region 2, an special case to look at is when $L_{1} / L_{2}=H_{1} / H_{2}>1$. If $L_{1} / L_{2}=H_{1} / H_{2}>1$ were an equilibrium, using equation (14) allows to obtain that in this case the relative price of housing in region 1 is:

$$
\frac{P_{h 1}}{P_{h 2}}=\frac{w_{1}}{w_{2}}
$$

Looking at equation (18) and the condition for $L_{1}>L_{2}$, we have $L_{1} / L_{2}=H_{1} / H_{2}>1$ cannot be an equilibrium.

From the simulations, two cases can be identified. On the one hand, when the dispersed equilibrium is stable, the simulations show that in equilibrium $L_{1} / L_{2}>H_{1} / H_{2}$. As Figures 3 and 4 show, this outcome arises when $\beta \varepsilon>1$, and for $\beta \varepsilon<1$ and domestic trade costs low enough. On the other hand, if the dispersed equilibrium is unstable, the opposite scenario takes place, with $L_{1} / L_{2}<H_{1} / H_{2}$ in equilibrium. This case takes place for $\beta \varepsilon<1$ and when domestic trade costs are high enough, or for intermediate domestic trade costs and high enough international trade costs.

\section{Trade liberalization}

In this section we focus on the effects of a reduction of import protection on the regional distribution of population in the dispersed stable equilibrium, when the distribution of housing is asymmetric.

The spatial distribution of economic activity between domestic regions results from the balance of centrifugal and centripetal forces. What are the potential effects that a reduction in $\tau$ may produce? With consumers looking for consuming a positive amount of every variety, we have that if the prices of manufactured varieties were the same, consumption of each variety will also be identical. However, under the existence of 
transports costs, the consumption of varieties produced domestically is larger, then, ceteris paribus, consumers have an incentive to live where the number of locally produced varieties is larger. As $\tau$ is reduced the there is a substitution effect in favour of imported varieties. With the consumption of imported varieties becoming more important, we may then expect the price of housing having a greater incidence on the decision where to live. So, as $\tau$ is reduced, populations should tend to distribute more in line with the supply of housing. As we can expect from the results of former section, the effects driven by changes in $\tau$ depend on the magnitude of the elasticity of substitution between manufactured varieties $(\varepsilon)$, as well as on the share of housing in the total expenditure $(\beta)$.

Figure 5 shows how the share of labour in Region 1 varies with the level of international trade costs $(\tau)$, for given values of domestic trade costs $(t)$, when Region 1 has $60 \%$ of the housing stock $\left(h_{1}=0.6\right)$. We focus on the cases where there is a single stable equilibrium with non-zero labour in Region 2 at all levels of trade protection.

When $\beta \varepsilon>1$ and inter-regional trade costs are high $(t=50$, Figure $5 \mathrm{~A})$, trade liberalization makes almost no difference to the distribution of labour, which is always extremely asymmetric $\left(s_{1}>0.97\right)$. For intermediate domestic transport costs $(t=2.3)$ the effects are quite large, with $s_{1}$ falling from 0.93 for $\tau=10$ to under 0.82 with free trade (Figure $5 \mathrm{~B})$. As $t$ becomes very low $(t=1.1)$ the effects are smaller because even at high values of $\tau s_{1}$ does not exceed 0.64 (Figure $5 \mathrm{C}$ ).

As said before, $\beta \varepsilon>1$ means consumers care more about the price of housing. In addition, if domestic transport costs are high enough, and under the assumption that $\tau>t$, the consumption of manufactured varieties produced by the ROW is almost nil. Then, changes in $\tau$ have almost no effect on the spatial distribution of firms between domestic regions. At the other extreme, if $t$ is low enough such that the consumption of varieties produced in the other domestic region is not too costly, the distribution of population across the two domestic regions is mainly driven by the cost of housing, then a reduction in $\tau$ has just a small effect on the distribution of population. It is when $t$ takes intermediate values that a change of it has a larger influence on $s_{1}$. Thus, for $\beta \varepsilon>1$ domestic trade costs have to fall within a certain range for international trade liberalization to have a substantial effect on the regional distribution of population in this model.

When $\beta \varepsilon<1$ consumers are more sensitive to the number of manufactured varieties, as well as housing has a more important role when deciding where to live. As imported varieties become cheaper due to the reduction in $\tau$, consumers substitute consumption of varieties produced by the ROW for local varieties. Then, a reduction of $\tau$ 
increases the relative importance of housing costs, inducing a migration from the larger to the smaller region (Figure 5D).

\section{Consumption bias}

A property of the manufactured composite index $m_{i}$ as defined by equation (2) is that, in the absence of transport costs $(t=\tau=1)$ consumption of manufactures is distributed proportionally to the number of varieties, that is, each consumer of region $i=1,2,3$ spends a proportion $n_{j} /\left(n_{1}+n_{2}+n_{3}\right)$ in goods produced in region $j=1,2,3$. If the domestic economy is small with respect to the ROW, this outcome means a disproportionately large import ratio. Table 1 shows the figures when the country represents only $1 \%$ of the world economy. Even with import protection, the participation of varieties produced by the ROW is still larger than tends to be observed in reality, even for extremely high levels of international trade costs.

This high import ratio can be corrected by introducing an asymmetry between the utility derived by consuming varieties produced by the home country and those produced by the ROW. There are at least two ways to introduce bias in consumers' preferences: by making use of the so-called "ideal variety" approach (Lancaster, 1979), or by keeping the "love for variety" formulation (Spence,1976; Dixit and Stiglitz, 1977) represented by equation (2) and introducing share parameters. We follow the second route.

Let us assume that for Regions $i=1,2$ the composite $m_{i}$ takes the following form:

$m_{i}=\left[\gamma \sum_{k \in n_{1}}\left(c(k)_{i}^{1}\right)^{\alpha}+\gamma \sum_{k \in n_{2}}\left(c(k)_{i}^{2}\right)^{\alpha}+(1-2 \gamma) \sum_{k \in n_{3}}\left(c(k)_{i}^{3}\right)^{\alpha}\right]^{\frac{1}{\alpha}}$

In equation (19) the parameter $1 / 3 \leq \gamma \leq 1 / 2$ is a measure of the relative preference for varieties produced domestically. If there is no bias in preferences $\gamma$ is equal to $1 / 3$, while if $\gamma>1 / 3$ consumers of Regions 1 and 2 have a bias in favor of domestic varieties (produced in either region). ${ }^{9}$ Similarly, for the ROW we have that $m_{3}$ takes the following form:

$m_{3}=\left[\theta \sum_{k \in n_{1}}\left(c(k)_{3}^{1}\right)^{\alpha}+\theta \sum_{k \in n_{2}}\left(c(k)_{3}^{2}\right)^{\alpha}+(1-2 \theta) \sum_{k \in n_{3}}\left(c(k)_{3}^{3}\right)^{\alpha}\right]^{\frac{1}{\alpha}}$

where $0 \leq \theta \leq 1 / 3$ measures the relative preference in favour of varieties produced in Regions 1 and 2. As before, consumers in the ROW do not make any distinction between varieties produced in regions 1 and $2 .^{10}$ 
Under the same assumptions on the producer side, as in the case without consumption bias, the scale of production is the same for all varieties, wherever they are produced. Also, the producer price of all varieties produced in the same region is identical

Table 2 summarizes the effects of introducing bias in preferences for low interregional transport costs $(t=1.1), h_{1}=0.6$ and $\beta \varepsilon<1$. As one would expect, when $\gamma$ is increased the share in manufacture consumption of varieties produced in the ROW falls. ${ }^{11}$

Table 3 shows the effect of $\gamma$ on the distribution of labour for the same parameter values. Before looking at the effects of changes in $\gamma$, let us point out that an increase in the relative size of the ROW induces a more symmetric distribution of population between the two domestic regions. The reason for this result is that as the ROW becomes larger, the consumption of domestic varieties becomes relatively less important than the consumption of foreign varieties, such that the incentive to agglomerate in order to reduce the burden of domestic trade costs is somewhat reduced. It still possible, however, to observe that in equilibrium population is more concentrated than housing $\left(s_{1}>h_{1}\right)$. As $\gamma$ increases, labour tends to become somewhat more concentrated in the region with more housing. Recall that, in Figure 4 , for $\beta \varepsilon<1$ and $t=2.3$, as $\tau$ increases, there is a stable equilibrium where Region 2 is empty of population and another stable equilibrium where Region 1 is either empty or has a very small population. As $\gamma$ increases from 1/3 towards $1 / 2$, the threshold combinations of $t$ and $\tau$ at which this occurs fall. In other words a dispersed equilibrium that is stable when $\gamma=1 / 3$ may not be so when $\gamma$ is higher.

A final issue is whether the presence of preference bias affects the rate at which regions' size converge as $\tau$ is reduced. Figure 6 maps the effects of reducing import protection for the same parameter values as in Tables 2 and 3 , and for four different values of $\gamma$. The effects increase as $\gamma$ rises from $1 / 3$ to 0.40 to 0.45 , but fall again for $\gamma=0.49$ (the same pattern is evident in Table 3 ). This happens because changes in trade protection matter very little both when consumers' desire for imported varieties shrinks to zero ( $\gamma$ approaches 1/2), and when imported varieties are so dominant in the consumption of manufactures that there is little incentive to be in the region that produces more manufactured varieties (the country is small compared with the ROW and $\gamma$ approaches $1 / 3)^{12}$

\section{Summary and conclusions}

Krugman and Livas Elizondo (1996) argue that the import substitution policy followed by many developing countries during most of the second half of the twentieth century 
generated, or at least intensified, a process of population and production concentration, leading to the emergence of industrial centres whose production was mainly intended for the domestic market. Can trade liberalization affect this geographical structure?

In order to throw some light on this question, we adapted Helpman's model to analyze the case where a domestic economy composed of two asymmetric regions reduces trade barriers on imports from the ROW. As is standard in NEG models, the agglomeration forces are generated through the inclusion of a sector that produces manufactured varieties under increasing returns to scale whose trade is subject to transport costs, and assuming labour is mobile between domestic regions (but not between countries). Dispersion forces are generated, as in Helpman (1998), by introducing a fixed regional supply of housing, which is not tradable between regions. This model has the advantage that it can easily incorporate asymmetries between the regions.

In this environment, and assuming an asymmetric distribution of housing, a reduction in trade costs on imports from the ROW tends to induce, ceteris paribus, a more equal distribution of population between the two domestic regions, so that manufactured production becomes less concentrated. This result is explained by the fact that, as imports becomes cheaper through trade liberalization, consumers seek to minimize the burden of housing costs, which are larger in the more populated region because the quantity of housing per capita is lower there (labour is always more unequally distributed than housing, except when there is no transport costs between domestic regions).

The picture is essentially the same when we allow for consumer preferences in the home country to be biased in favour of domestically produced varieties. In the presence of consumption biases, the effect of trade liberalization on regions' size tends to be larger (when $\beta \varepsilon>1$ ), except in the case of extreme preferences for home-produced manufactures.

\section{References}

[1] Alonso-Villar, O. (2001), 'Large metropolises in the Third World: an explanation'. Urban Studies, 38, 1359-1371.

[2] Brakman, S.; Garretsen, H. and Schramm, M. (2002), 'The Spatial Distribution of Wages and Employment: Testing the Helpman-Hanson model for Germany'. Mimeo.

[3] Crozet, M. and Koening-Souberyarn, P. (2002), 'Trade liberalization and the internal geography of countries'. CREST Discussion Paper, 2002-37.

[4] Dixit, A. and Stiglitz, J. E. (1977), 'Monopolistic competition and optimum product diversity'. American Economic Review, 67, 297-308.

[5] Fujita, M, Krugman, P. and Venables, A. (1999), The spatial economy. Cities, regions and international trade. MIT Press. 
[6] Hanson, G. H. (1994), 'Localization economies, vertical organization, and trade'. NBER Working Paper, 4744.

[7] Hanson, G. H. (2005), 'Market potential, increasing returns and geographic concentration'. Journal of International Economics, 67, 1 -24.

[8] Helpman, E. (1998), 'The size of regions', in: D. Pines, E. Sadka and I. Zilcha (eds.), Topics in Public Economics. Theoretical and Applied Analysis, 31-54. Cambridge University Press.

[9] Krugman, P (1991), 'Increasing returns and economic geography'. Journal of Political Economy, 99, 483-499.

[10] Krugman, P. and Livas Elizondo, R. (1996), 'Trade policy and Third World metropolis'. Journal of Development Economics, 49, 137-150.

[11] Krugman, P. and Venables, A. (1995), 'Globalization and the inequality of nations'. Quarterly Journal of Economics, 110, 857-880.

[12] Krugman, P. and Venables, A. (1996), 'Integration, specialisation, and adjustment'. European Economic Review, 40, 959-967.

[13] Lancaster, K. (1979), Variety, equity and efficiency. Columbia University Press, New York.

[14] Mion, G. (2004), 'Spatial externalities and empirical analysis: the case of Italy'. Journal of Urban Economics, 56, 97-118.

[15] Monfort, P. and Nicolini, R. (2000), 'Regional convergence and international integration'. Journal of Urban Economics, 48, 286-306.

[16] Murata, Y. and Thisse, J-F. (2005), 'A simple model of economic geography à la Helpman-Tabuchi'. Journal of Urban Economics, 58, 137-155.

[17] Paluzie, E. (2001), 'Trade policies and regional inequalities'. Papers in Regional Science, $80,67-85$.

[18] Spence, M. E. (1976), 'Product selection, fixed costs, and monopolistic competition'. Review of Economic Studies, 43, 217-236.

[19] Venables, A. J. (1996), 'Equilibrium locations of vertically linked industries'. International Economic Review, 37, 341-359. 
$t=1.1$

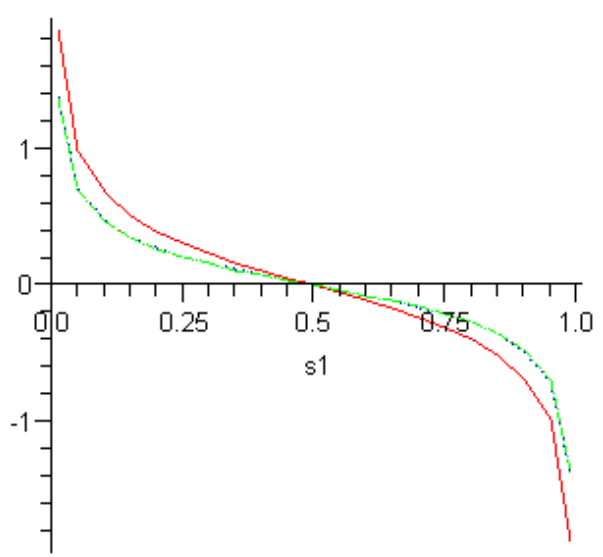

$-\quad \tan =1.1$

…....... tau $=5$

- . - . tau=100

tau $=1000$
$T=2.3$

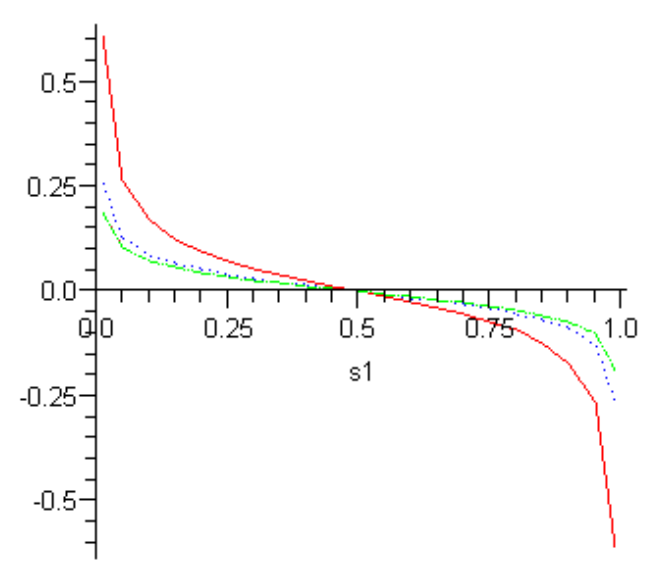

$\operatorname{tau}=2.3$

tau $=5$

. . . . tau=100

tau $=1500$ $t=50$

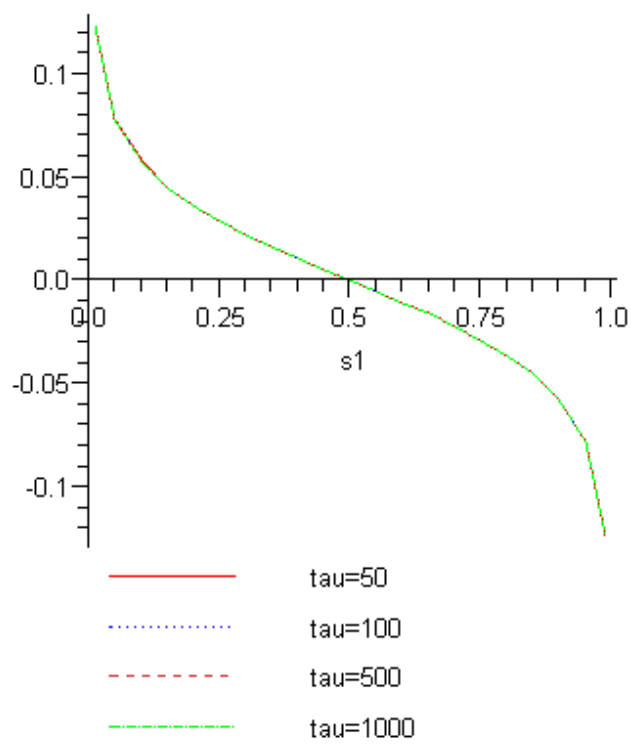

Figure 1: A Symmetrical Distribution of Housing $(\beta \varepsilon>1)$. Vertical axis: real income in Region 1 minus real income in Region 2 (y). A positive (negative) value implies an incentive to migrate to region 1 (2). Horizontal axis: share of population in Region $1\left(s_{1}\right)$. If $y>0(<0), s_{1}$ tends to increase (decrease) over time, so a stable interior equilibrium requires a downward sloping curve. Parameter values: $\beta=0.2, \varepsilon=5.5, h_{1}=1 / 2$. 
$t=1.1$

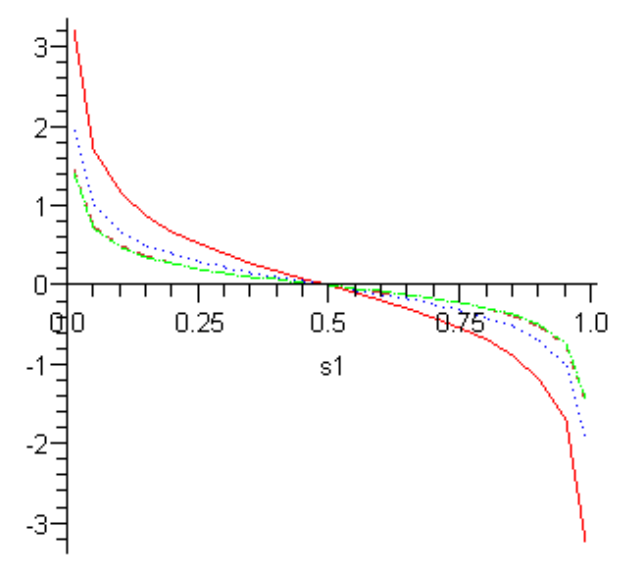

tau=1.1

tau $=5$

tau=100

tau $=10000$ $t=2.3$

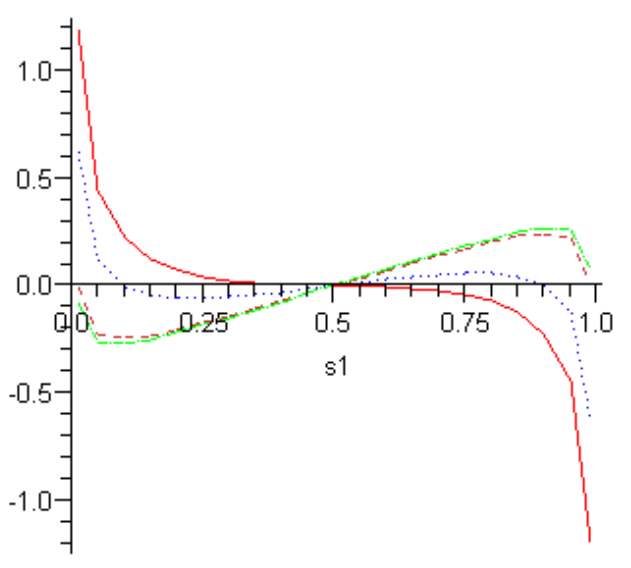

$\mathrm{tau}=2.3$

tau=5

tau $=100$

tau=10000 $t=50$

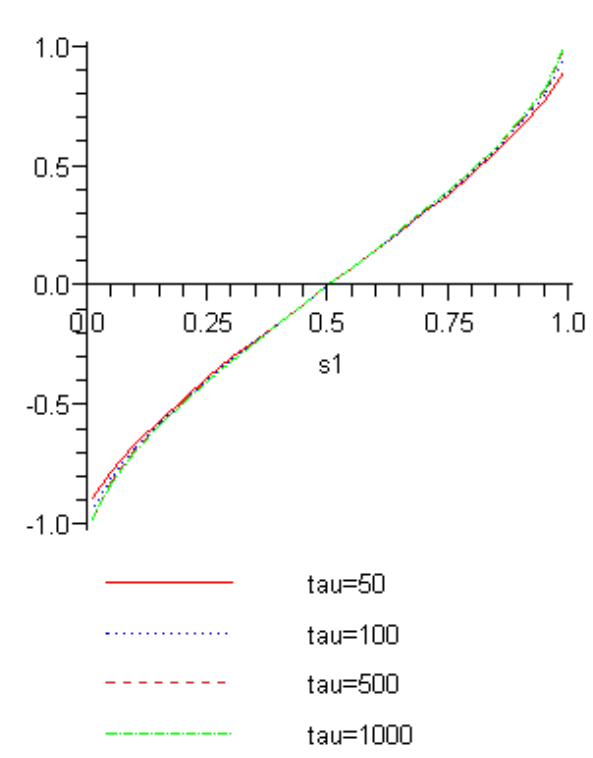

Figure 2: A Symmetrical Distribution of Housing $(\beta \varepsilon<1)$. Vertical axis: real income in Region 1 minus real income in Region 2 (y). A positive (negative) value implies an incentive to migrate to region 1 (2). Horizontal axis: share of population in Region $1\left(s_{1}\right)$. If $y>0(<0), s_{1}$ tends to increase (decrease) over time, so a stable interior equilibrium requires a downward sloping curve. Parameter values: $\beta=0.2, \varepsilon=2.2, h_{1}=1 / 2$. 
$t=1.1$

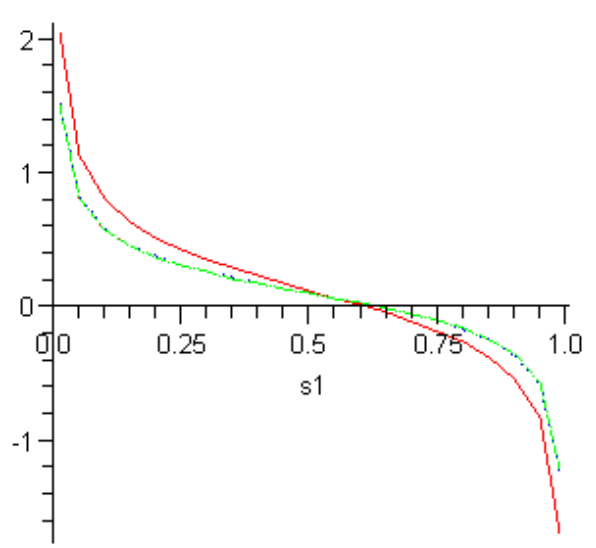

$-$

…....... tau $=5$

- . . . tau $=100$

tau $=1000$ $t=2.3$

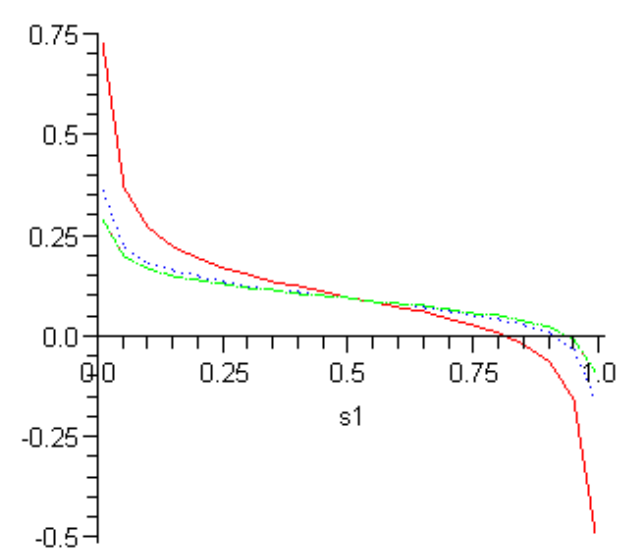

$\operatorname{tau}=2.3$

tau $=5$

tau $=100$

tau $=1500$ $t=50$

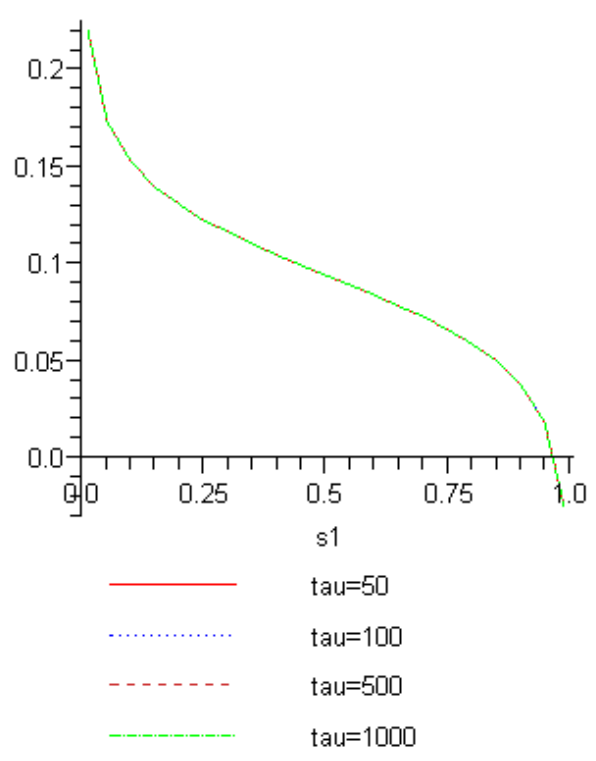

Figure 3: Asymmetric Distribution of Housing $(\beta \varepsilon>1)$. Vertical axis: real income in Region 1 minus real income in Region 2 (y). A positive (negative) value implies an incentive to migrate to region 1 (2). Horizontal axis: share of population in Region $1\left(s_{1}\right)$. If $y>0(<0), s_{1}$ tends to increase (decrease) over time, so a stable interior equilibrium requires a downward sloping curve. Parameter values: $\beta=0.2, \varepsilon=5.5, h_{1}=0.6$ 
$t=1.1$

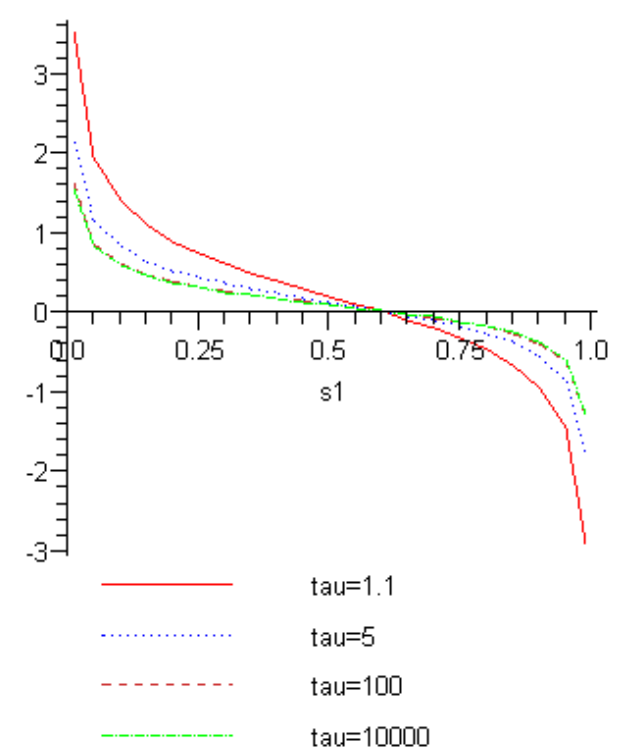

$t=2.3$

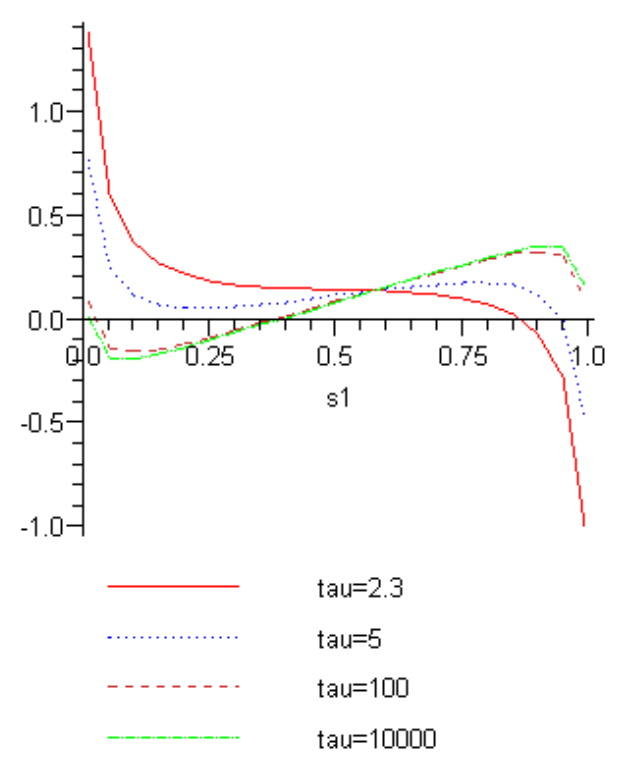

$t=50$

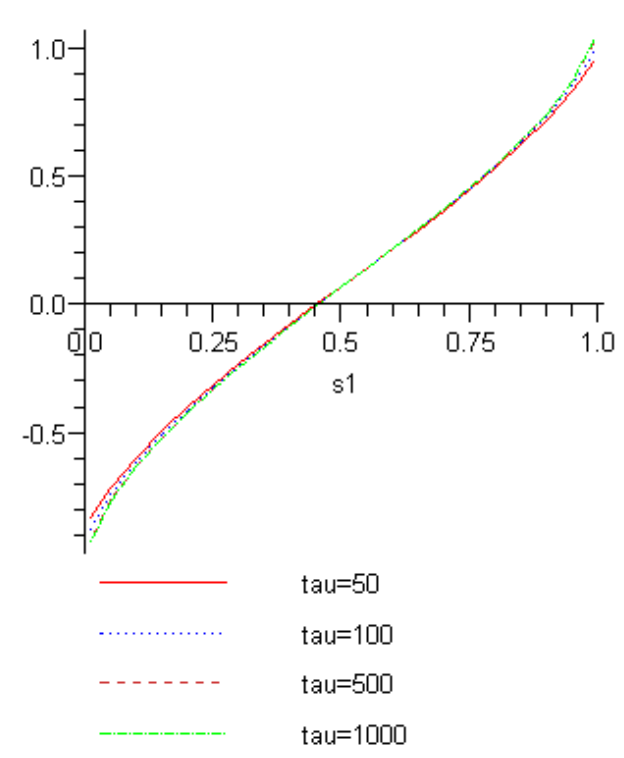

Figure 4: Asymmetric Distribution of Housing $(\beta \varepsilon<1)$. Vertical axis: real income in Region 1 minus real income in Region 2 (y). A positive (negative) value implies an incentive to migrate to region 1 (2). Horizontal axis: share of population in Region $1\left(s_{1}\right)$. If $y>0(<0), s_{1}$ tends to increase (decrease) over time, so a stable interior equilibrium requires a downward sloping curve. Parameter values: $\beta=0.2, \varepsilon=2.2, h_{1}=0.6$. 
A) $\beta \varepsilon>1, t=50(*)$

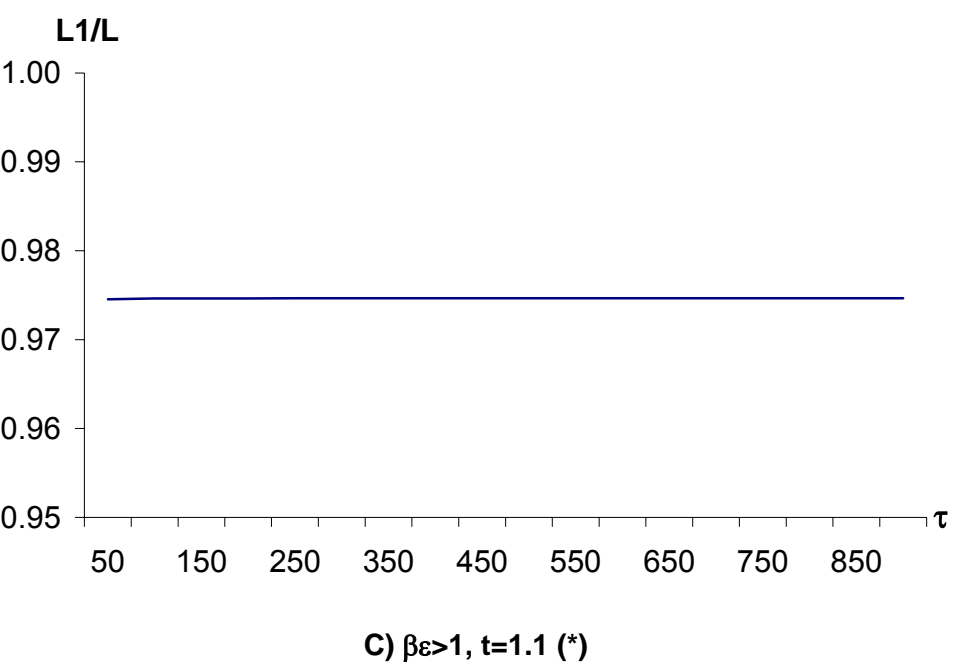

\section{L1/L}

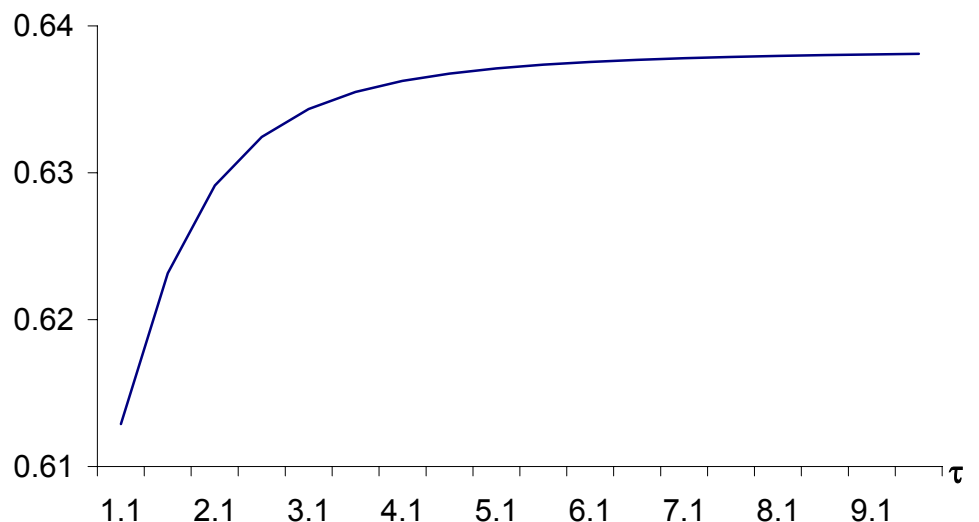

B) $\beta \varepsilon>1, t=2.3\left(^{*}\right)$

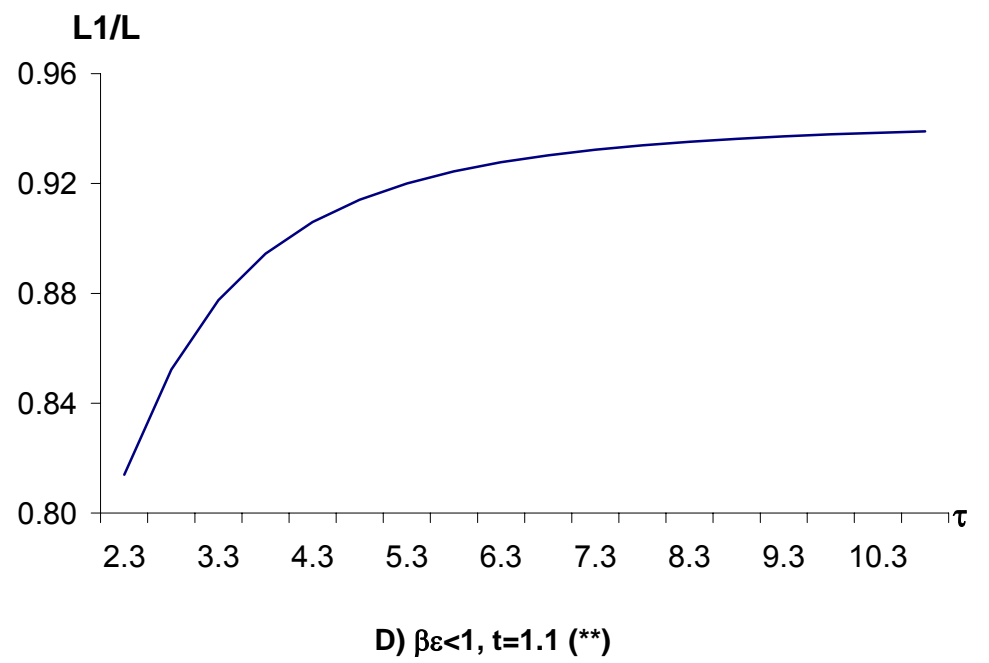

L1/L

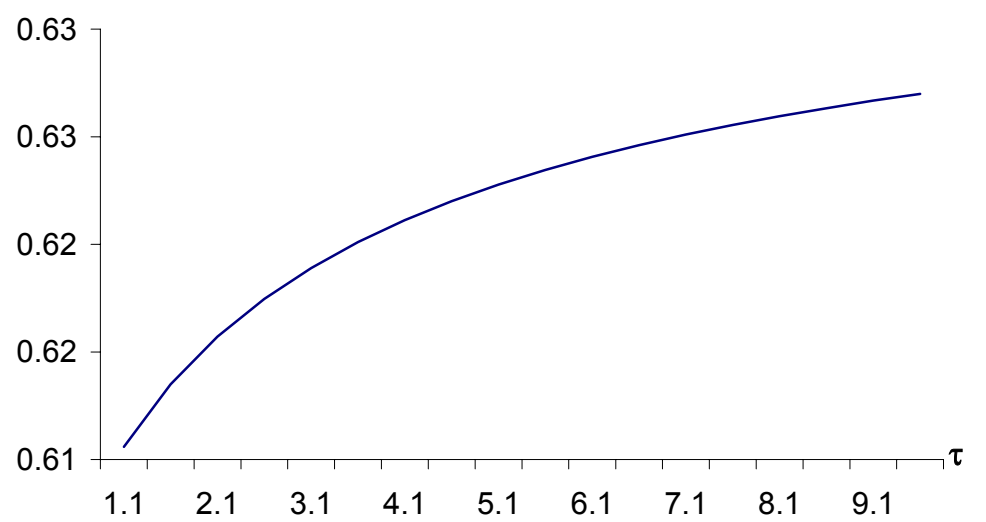

Figure 5. International trade costs and regions' sizes. Asymmetric distribution of housing. Vertical axis: share of population in Region 1 ( $\left.s_{1}\right)$. Horizontal axis: international transport costs $(\tau)$. Parameter values: $h_{1}=0.6 ; \beta=0.2 ; \quad \varepsilon=2.2\left({ }^{*}\right) ; \varepsilon=5.5\left(^{* *}\right)$. 


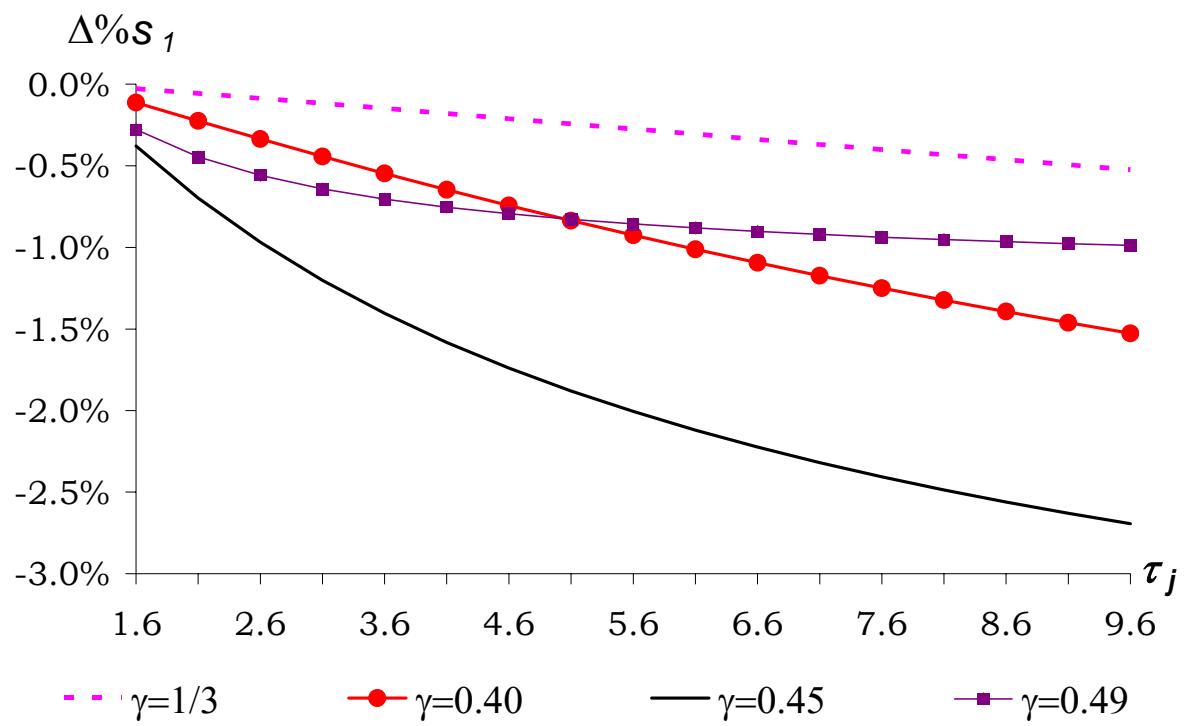

Figure 6: percentage variation in $s_{1}$ as $\tau$ is reduced from $\tau_{j}$ to $\tau=1.1$.

$\beta \varepsilon<1 ; \beta=0.2, \varepsilon=2.2, h_{1}=0.6, t=1.1$. 
Table 1

Region 1: manufactured expenditure shares

$\beta \varepsilon<1(*), h_{1}=0.6, t=1.1, L_{3}=99$

\begin{tabular}{|c|c|c|c|c|c|c|}
\hline$\tau$ & $\mathbf{1 . 1}$ & $\mathbf{2 . 1}$ & $\mathbf{3 . 1}$ & $\mathbf{4 . 1}$ & $\mathbf{5 . 1}$ & $\mathbf{6 . 1}$ \\
\hline Domestic varieties (R1+R2) & $1.1 \%$ & $2.3 \%$ & $3.5 \%$ & $4.8 \%$ & $6.2 \%$ & $7.5 \%$ \\
\hline Imports from Region 3 & $98.9 \%$ & $97.7 \%$ & $96.5 \%$ & $95.2 \%$ & $93.8 \%$ & $92.5 \%$ \\
\hline
\end{tabular}

Note: when $t=\tau=1$, the shares are, respectively, $1 \%$ and $99 \%$.

$\left.{ }^{*}\right) \beta=0.2, \varepsilon=2.2$. When $\beta \varepsilon>1$ the share of foreign varieties reduces appreciably.

Table 2

Region 1: manufactured expenditure shares

$\beta \varepsilon<1(*), h_{1}=0.6, t=1.1, L_{3}=99$

\begin{tabular}{|l|r|r|r|r|r|}
\hline \multicolumn{1}{|c|}{$\tau$} & \multicolumn{1}{|c|}{$\mathbf{1 . 1}$} & \multicolumn{1}{|c|}{$\mathbf{3 . 1}$} & \multicolumn{1}{|c|}{$\mathbf{5 . 1}$} & \multicolumn{1}{|c|}{$\mathbf{7 . 1}$} & \multicolumn{1}{c|}{$\mathbf{9 . 1}$} \\
\hline$\gamma=\mathbf{1 / 3}$ & & & & & \\
Domestic varieties (R1+R2) & $1.1 \%$ & $3.5 \%$ & $6.2 \%$ & $8.8 \%$ & $11.3 \%$ \\
Imports from Region 3 & $98.9 \%$ & $96.5 \%$ & $93.8 \%$ & $91.2 \%$ & $88.7 \%$ \\
\hline$\gamma=\mathbf{0 . 4 0}$ & & & & & \\
Domestic varieties (R1+R2) & $4.6 \%$ & $13.7 \%$ & $21.5 \%$ & $28.0 \%$ & $33.4 \%$ \\
Imports from Region 3 & $95.4 \%$ & $86.3 \%$ & $78.5 \%$ & $72.0 \%$ & $66.6 \%$ \\
\hline$\gamma=$ 0.45 & & & & & \\
Domestic varieties (R1+R2) & $20.6 \%$ & $43.0 \%$ & $54.7 \%$ & $62.0 \%$ & $67.0 \%$ \\
Imports from Region 3 & $79.4 \%$ & $57.0 \%$ & $45.3 \%$ & $38.0 \%$ & $33.0 \%$ \\
\hline$\gamma=0.49$ & & & & & \\
Domestic varieties (R1+R2) & $82.6 \%$ & $91.7 \%$ & $94.2 \%$ & $95.5 \%$ & $96.3 \%$ \\
Imports from Region 3 & $17.4 \%$ & $8.3 \%$ & $5.8 \%$ & $4.5 \%$ & $3.7 \%$ \\
\hline
\end{tabular}

(*) $\beta=0.2, \varepsilon=2.2$

Table 3

Model with consumption bias: $\mathrm{L}_{1} / \mathrm{L}$

$\beta \varepsilon<1(*), h_{1}=0.6, t=1.1, L_{3}=99$

\begin{tabular}{|c|c|c|c|c|c|}
\hline$\tau$ & 1.1 & 3.1 & 5.1 & 7.1 & 9.1 \\
\hline$\gamma=\mathbf{1 / 3}$ & 0.6003 & 0.6010 & 0.6018 & 0.6025 & 0.6033 \\
$\gamma=\mathbf{0 . 4 0}$ & 0.6013 & 0.6040 & 0.6064 & 0.6085 & 0.6102 \\
$\gamma=\mathbf{0 . 4 5}$ & 0.6061 & 0.6135 & 0.6177 & 0.6205 & 0.6225 \\
$\gamma=\mathbf{0 . 4 9}$ & 0.6290 & 0.6330 & 0.6342 & 0.6348 & 0.6352 \\
\hline
\end{tabular}

(*) $\beta=0.2, \varepsilon=2.2$ 


\footnotetext{
${ }^{1}$ The notion of iceberg costs means that for each unit of an imported variety that is consumed, more than 1 unit must be shipped from the exporting region.

${ }^{2}$ Here we follow Krugman and Livas Elizondo (1996). This asymmetry in international transport costs is innocuous to the results of the model.

${ }^{3} \mathrm{t}(\tau)$ is the quantity of each variety that must be shipped by a domestic (foreign) firm for 1 unit to arrive to the importing region.

${ }^{4}$ As Krugman and Livas Elizondo (1996) point out, $t$ should be interpreted as "natural" transport costs, while $\tau$ is a combination of natural transport costs and artificial trade barriers.

${ }^{5}$ Helpman (1998) assumes the stock of housing is evenly owned by all population. The assumption of absentee landlords is innocuous to the results of the model but it simplifies greatly the expression for regions' expenditure.

${ }^{6}$ Because of the circularity introduced by the assumption that labour migrates in response to differences in real wages is not possible to obtain closed forms for the variables of interest. The analysis of sections 3,4 and 5 is carried out through the use of numerical simulations.

${ }^{7}$ To be more precise, under a Cobb-Douglas utility function, we have that unless domestic transport costs are infinity, no region can ever be empty because the price of housing would be zero and any migrant could reach an infinite level of utility when first moving to this region.

${ }^{8}$ By free trade we refer to the case when domestic and international transport costs are the same.

${ }^{9}$ If $\gamma=1 / 2$ we have a closed economy, with the results resembling those of Helpman (1998).

${ }^{10}$ By restricting the possible values taken by $\gamma$ and $\theta$, such that $\gamma \geq 1 / 3$ and $\theta \leq 1 / 3$, we rule out the possibility of consumers having a greater preference for foreign varieties.

${ }^{11}$ In all simulations it is assumed that consumers of Region 3 have no bias in preferences

$(\theta=1 / 3)$.

${ }^{12}$ The same pattern arises if we look at level changes instead of percentage changes.
} 